\title{
Peer and Self Evaluation for Cooperative Learning in Interpreting Course
}

\author{
Yan Cao \\ Foreign Language School \\ East China University of Political Science and Law \\ Shanghai, China \\ Email: zoe cao29@163.com
}

\begin{abstract}
Due to limited class hours and heavy workload, the teacher could not ensure each student enough opportunities for interpreting practice and feedback. This study seeks to address this problem by embedding peer and self evaluation in cooperative learning activities of the interpreting course. To improve the effect of peer and self-evaluation, the teacher needs to model the process of evaluation and create a supportive culture. It is found that peer and self evaluation not only increase students' responsibility and learner autonomy, but also reduce teachers' marking load and improve working efficiency.
\end{abstract}

Keywords-peer and self evaluation; feedback; cooperative learning; interpreting course

\section{INTRODUCTION}

In the interpreting course, interpreting exercises are usually organized by the teacher, in which students listen to a speech, and interpret it into the target language segment by segment. After listening to each segment, the teacher picks one student to interpret in front of the whole class; or record everyone's interpreting, then chooses one or two to listen to and points out the possible mistakes or gives suggestions about interpreting strategies. Students benefit a lot from teachers' feedback. However, the problem with the traditional classroom teaching of interpreting is that only a few students have the opportunity to get feedback from the teacher during the limited class hours. Constrained with heavy workloads, it is barely impossible for the teacher to give each student detailed feedback and instruction. Peer and self evaluation are important aspects of evaluation for learning practice. Assessing their own work or that of others can help students develop their understanding of learning objectives and success criteria. This study seeks to address this problem by embedding peer and self evaluation in cooperative learning activities of interpreting course.

\section{Theoretical Background of PeER AND SelF EVALUATION}

Assessment typically frames how higher education students learn because it provides the clearest indication of what the institution gives priority to (Boud, 2007). It is an integral part of the learning process. When teachers share with their students the process of assessment, giving up control, sharing power and leading students to take on the authority to assess themselves, the professional judgment of both is enhanced. Assessment becomes not something done to students. It becomes an activity done with students. (Brew,1999: 169)
Peer and self assessment offer students the opportunity to be actively involved in the assessing process.

\section{A. Definition of self evaluation}

According to Boud (1995), all assessment including self-assessment comprises two main elements - making decisions about the standards of performance expected and then making judgments about the quality of the performance in relation to these standards. When self-assessment is introduced, it should ideally involve students in both of these aspects.

Andrade and Du (2007) provide a definition of self-assessment focusing on the formative learning it can promote:

Self-assessment is a process of formative assessment during which students reflect on and evaluate the quality of their work and their learning, judge the degree to which they reflect explicitly stated goals or criteria, identify strengths and weaknesses in their work, and revise accordingly (2007: 160).

\section{B. Definition of peer evaluation}

Though there are many variants of peer assessment, essentially it means students providing feedback to other students on the quality of their work. Falchikov provide a definition of peer-assessment.

Peer assessment requires students to provide either feedback or grades (or both) to their peers on a product or a performance, based on the criteria of excellence for that product or event which students may have been involved in determining" (2007: 132).

Whatever form of peer-assessment is used, the method should allow students to practice making reasonable judgements about the extent to which their peers have achieved expected outcomes (Falchikov, 2007).

\section{Benefits of peer and self evaluation}

Self evaluation with its emphasis on student responsibility and making judgments is "a necessary skill for lifelong learning" (Boud, 1995: 11). Additionally, the self-assessment process can help "to prepare students not just to solve the problems we already know the answer to, but to solve problems we cannot at the moment even conceive" (Brew, 1995: 57).

Peer evaluation helps to lessen the power imbalance between teachers and students and can enhance the students' status in the learning process. Students engaged in commenting on the work of others can enhance their own capacity for judgment and making intellectual choices. 
Studies show that peer and self evaluation can involve students in critical reflection, which is the key to successful autonomous learning (Boud, 1995; Carr, 2002). Most practitioners aspire to have independent learners who possess a clear understanding of their learning journey and can actively expand their own skills and knowledge, impacting on their progress. Their research concludes that peer and self assessment is one route to achieving this:

Self and peer assessment are important aspects of assessment for learning practice... Assessing their own work or that of others can help pupils develop their understanding of learning objectives and success criteria. Research has shown that pupils make more progress when they are actively involved in their own learning and assessment. (NFER 2012)

Shirley Clarke (2008) found that "looking at the work of others can ...help pupils to understand the different approaches they could have taken...that there are different ways of achieving success."

\section{Developing Peer and Self Evaluation in INTERPRETING COURSE}

Cooperative learning activities can be more successful when students are involved in developing peer and self evaluation process. The development of effective self and peer evaluation takes considerable time and effort. However, once fully embedded into the interpreting course, they can be particularly effective in motivating students to move forward in their interpreting learning.

\section{A. Peer evaluation in interpreting course}

It is recommended that peer evaluation should be introduced first, because it is relatively easier to find errors and mistakes in others' performance (Liu \& Carless, 2006). In the cooperative learning activity for the interpreting course, students work in groups of three. One student plays the role of a speaker; another student plays the role of an interpreter; a third one is the reviewer. The speaker gives a segment of the speech and pauses for the interpreter interprets the meaning into the target language. After the speaker and the interpreter finishes the presentation, the assessor analyze and comment on the interpreter's performance, pointing out possible errors and mistakes. Apart from verbal comment, the assessor has to fill a peer evaluation form, which includes rating instruments and checklists of accuracy, fluency, articulacy and timing. Group members take these roles by rotation.

In order to ensure the effectiveness of peer evaluation, the teacher should make the rules for giving feedback explicit and ensure that students understand and follow these rules when working in groups. When evaluating others' interpreting performance, students are encouraged to identify successful features and respect the work of others. When suggesting improvements, students should think about the learning objective of the interpreting course and criteria for successful interpretation.

\section{B. Self evaluation in interpreting course}

Looking at the work of others can also help students to understand the different approaches they could have taken and to appreciate that there are different ways of achieving success (Kowalke, 1998). Having evaluated the work of others, students may find it easier to identify weaknesses in their own performance and to see how they can make progress in interpreting. Students are asked to record their own interpreting exercises and fill in a self evaluation form after listening to the recorded performance. Marks are given in three aspects: content, language and delivery. Students should be encouraged to reflect on their own development and progress, comparing their current work with that produced previously and with their personal targets.

\section{Modeling the process of evaluation}

Students need to be supported in giving effective feedback. Teachers should help them to understand the different types of feedback that can be given and how each type can help others to improve their work. Therefore, the starting point for introducing peer evaluation is for teachers to act as a role model, and demonstrate and explain how evaluation should be done. For example, the teacher may show students how to give constructive feedback (detailed comments, objective focus, etc.) both verbally and through marking. Showing examples of work that do and do not meet the success criteria can help students to better understand what is required and to reflect on the things they need to do in order to improve.

Teachers can use examples of interpreting from anonymous students, modeling the type of constructive feedback that might be given or providing a list of questions that students might ask. This helps students to become accustomed to the process before they are asked to evaluate and comment on the work of their classmates.

\section{Creating a supportive culture for evaluation}

Self and peer evaluation require a classroom culture or atmosphere where errors or mistakes are valued as learning opportunities and admitting to not understanding something or not capable of doing something is acceptable. Group members act as critical partners, critiquing the work of others in a way that both supports and challenges them and facilitates their future progress. In order for such a culture to thrive, teachers must be able to show that they too can make mistakes. Teachers may cite examples to show that even experienced interpreters may make mistakes or come across difficulties in practice, thus making students realize that making mistakes is a process of making progress.

\section{IMPLEMENTING PEeR AND SElf EVAluation IN INTERPRETING COURSE}

\section{A. Principles of peer and self evaluation}

Both the teacher and students should be clear about the purpose of using self and peer evaluation in interpreting course, which is to improve students' interpreting competence through more practice and enhance students' reflection on their practice. Peer and self evaluation processes require students to take responsibility for their actions, and encourage them to take a reflective approach to learning, providing, seeking and ustilizing feedback.

The size of groups has to be three or four. In that situation, students have an opportunity to compare their fellow students' remarks, and to determine their relevance. A group of two students is too small, because of the risk that bias may exist in their evaluation. Group members take turns to play different roles in cooperative learning activities, as it is clear that the assessor will in turn be the assessee, which make it easier to exchange opinions and give constructive feedback.

\section{B. Careful planning of cooperative learning events}

To many students, assessment is a job for the teacher. But gradually they are likely to recognize the benefits of peer and self evaluation to their learning and skill development as they are involved in more cooperative learning events. Andrade and Du (2007) found that students' attitudes toward assessment became more positive as they gained experience with it. The students' experiences were more positive if teachers provided clear articulation of assessment criteria and rubrics. More positive responses to the use of peer and self assessment are likely when the teacher's expectations are clear and when the students have received systematic practice. Initial efforts take time and teacher support. Self and peer evaluation procedures should be subject to particularly careful monitoring and evaluation from the teacher. After utilizing peer and self evaluation 
in cooperative learning events for a period of time, the teacher should allow fading of support so that students may move nearer towards assessment autonomy.

\section{EfFect of Peer and Self Evaluation}

Peer and self evaluation encourage students to reflect on their role and contribution to the process of the group work. In this study, we engaged students in the process by making peer and self evaluation an integral part of the cooperative learning activity and used the results of peer and self evaluation as important sources of information. After implementing peer and self evaluation for one semester, we had focus group interview with students of the interpreting course to find out the effect. A majority of students agreed that they had had more opportunities to practice assessment procedures and gained critical thinking skills. Some students found it very motivating because they had a larger audience for their interpreting performance. The result of the interview also showed peer and self evaluation made it easier for students to contribute to target-setting. They had a better understanding of the criteria for interpreting through evaluating others' and their own work. When asked about the form of evaluation, students said verbal peer evaluation worked better than written feedback.

Our study shows, when properly designed and managed, peer and self evaluation have the potential to increase student responsibility and learner autonomy. Assessing their own performance and those of other group members could lead to a more advanced and deeper understanding of the subject matter, skills and processes. In fact, peer and self evaluation lift the role and status of the student, encouraging a deeper approach to learning. Developing self and peer evaluation skills are a valuable investment for the long-term future of students, helping them become autonomous learners who are capable of lifelong learning. Peer and self evaluation promote meta-cognition in learners in that it helps them develop a deeper awareness of how they learn and promotes better learning. For teachers, when operating successfully, peer and self evaluation can reduce a teacher's marking load and improve working efficiency.

\section{Potential Problems with Peer and Self Evaluation}

A potential danger of students evaluating the performance of each other is that some students may be excessively or insufficiently challenged. Some of these students may even lose motivation for further study. Therefore, it is important that students understand that everyone's learning process may be different. It is also important that students can admit to areas of weakness without risk to their self-esteem. Asking students to word their suggestions and comments positively may lessen this danger.

Another common problem is that bias exists in students' peer and self evaluation. On the one hand, friendship may influence peer evaluation. When evaluating others' performance, many students are more lenient, avoiding the use of the lowest or highest rating categories. Some students tend to award everyone the same mark, while others are reluctant to make judgments regarding their peers. On the other hand, self evaluation has a risk of being perceived as a process of presenting inflated grades and being unreliable. To achieve better quality peer and self evaluation, the teacher need to inform students their bias patterns and help them develop a better understanding of their own subjectivity and judgment as well as better quality assessment criteria. Strictly adhering to the same criteria for evaluation may help to reduce bias.

A third problem with peer and self evaluation is that students may have little exposure to different forms of assessment, and as a result they may lack the necessary skills to effectively manage self and peer evaluation. Demonstrating the process of evaluation by the teacher can be effective in solving this problem.

\section{CONCLUSION}

In this study, we try to use both peer and self evaluation as one useful technique for learning interpreting. Embedding peer and self evaluation in cooperative learning activities of interpreting course can give students more opportunities for doing interpreting practice and getting feedback. To improve the quality of peer and self evaluation, the teacher should provide guidelines in advance as to what students are expected to focus on in their evaluation. Modeling of evaluation process through whole class evaluation of a model example can help students identify areas of success, leading to a clear set of success criteria and expectations. Creating a supportive culture is also necessary to ensure the success of peer and self evaluation. Peer evaluation should be introduced first and only moving on to self evaluation when students are fully comfortable with the former. Interviews with students show a majority of students agree that they benefit through taking part in the peer and self evaluation. Peer and self evaluation help students change from passive learner to active learner and assessor, and increase student responsibility and learner autonomy. Peer and self evaluation also contribute to the development of self-appraisal, evaluative, analytical, critical and reflective skills. Potential problems with peer and self evaluation include losing motivation, bias and lack of evaluation skills, which could be solved through taking relevant measures.

\section{REFERENCES}

[1] H. Andrade, \& Y. Du, "Student responses to criteria-referenced self-Assessment," Assessment and Evaluation in Higher Education, 2007, 32 (2), pp. 159-181.

[2] D. Boud, "Enhancing Learning Through Self Assessment," Philadephia: Kogan Page, 1995.

[3] A. Brew, "What is the scope of self assessment?" In D.Boud, Enhancing learning through self-assessment, London: Kogan Page, 1995 , pp.48-63.

[4] C. Carr, Sonya, "Self-evaluation: Involving Students in Their Own Learning," Reading \& Writing Quarterly, 2002, 18: pp. 195-199.

[5] L. Kowalke "Student Self-Assessment: Thinking about Thinking," Methods that Matter: Six Structures for Best Practice Classrooms. Ed. Harvey Daniels and Marilyn Bizar. York, Maine: Stenhouse Publishers. 1998, pp. 233-242.

[6] N. F. Liu and D. Carless, "Peer feedback: the learning element of peer assessment," Teaching in Higher Education, 2006, 11(3): pp. 279-290.

[7] H. McMillan, James, "Classroom Assessment: Principles and Practice for Effective Instruction,” Boston: Allyn and Bacon, 2001.

[8] NFER - "Getting to grips with assessment: Primary," (updated 2012) Retrieved from https://www.nfer.ac.uk/pdf/getting-to-grips-with-assessment-4.pdf

[9] J. Simmons, "Responders are Taught, not Born," Journal of Adolescent \& Adult Literacy, 2003, 46: pp. 684-693.

[10] C. Shirley, "Active learning through formative assessment," TRANS-ATLANTIC PUBNS, 2008. 